\title{
MULTIPLE SEPARATION THEOREMS
}

\author{
ASHOK MAITRA
}

(Communicated by Andreas R. Blass)

ABSTRACT. We prove multiple separation theorems for duals of Spector pointclasses.

\section{INTRODUCTION}

The aim of this article is to prove an effective version of the multiple separation theorems of classical descriptive set theory (see Kuratowski [3, pp. 510512] or Liapunov [4]). The effective version is formulated for duals of Spector pointclasses closed under $\exists^{\omega^{\omega}}$ of $\forall^{\omega^{\omega}}$ and is proved by exploiting the pre-wellordering theory of such pointclasses, as described in Moschovakis [5, Chapter 4]. By specializing the bold-face version of our result to the case where the Spector pointclass is a member of the analytic hierarchy, we recover the classical results in $[3,4]$.

In the classical theory, P. S. Novikov was the first to formulate and establish multiple separation theorems. He did this at the first two levels of the projective hierarchy for the operation of countable intersection (see [3, pp. 510-512]). This was later generalized to $\delta$-s operations of greater complexity by Liapunov in a sequence of papers culminating with [4]. More recent treatments of the problem are to be found in Davies et al. [1] and Saint-Pierre [6].

The next section is devoted to preliminaries, and the main result will be proved in $\S 3$.

\section{Preliminaries}

The set of natural numbers will be denoted by $\omega$, and $P(\omega)$ will denote the powerset of $\omega$. For any nonempty set $N \subseteq P(\omega)-\{\varnothing\}$, the $\delta$-s operation $\Phi_{N}$ on a sequence of sets is defined as

$$
\Phi_{N}\left(E_{0}, E_{1}, \ldots\right)=\bigcup_{\eta \in N} \bigcap_{n \in \eta} E_{n}
$$

or

$$
x \in \Phi_{N}\left(E_{0}, E_{1}, \ldots\right) \leftrightarrow(\exists \eta \in N)(\forall n \in \eta)\left(x \in E_{n}\right) .
$$

The set $N$ is called the base of the $\delta$-s operation $\Phi_{N}$.

Received by the editors April 15, 1992 and, in revised form, May 26, 1992.

1991 Mathematics Subject Classification. Primary 03E15; Secondary 04A15.

Key words and phrases. Separation theorems, Spector pointclass, projective hierarchy. 
A set $N \subseteq P(\omega)-\{\varnothing\}$ is said to be complete if

$$
\eta \in N \& \eta \subseteq \eta^{\prime} \subseteq \omega \rightarrow \eta^{\prime} \in N
$$

The base of a $\delta$-s operation can always be chosen to be complete. Indeed, if $\Phi_{N}$ is a $\delta$-s operation, then set $\bar{N}=\Phi_{N}\left(D_{0}, D_{1}, \ldots\right)$ where $D_{n}=\{\eta \subseteq \omega: n \in \eta\}$, $n \geq 0$.

It is easy to verify that $\bar{N}$ is complete and that $\Phi_{N}=\Phi_{\bar{N}}$. For further information on $\delta$-s operations, the reader is referred to Kantorovich and Livenson [2].

Following Moschovakis [5, p. 207], we say that $\Gamma$ is a Spector pointclass if

(a) $\Gamma$ contains all semirecursive pointsets and is closed under $\vee, \&, \exists^{\omega}$, and $\forall^{\omega}$

(b) $\Gamma$ is $\omega$-parametrized;

(c) $\Gamma$ is normed; and

(d) $\Gamma$ has the substitution property.

The dual of a pointclass $\Gamma$ will be denoted by $\neg \Gamma$, and we write $\Delta$ for $\Gamma \cap 7 \Gamma$. If $\Gamma$ is a pointclass and $X=\omega^{k} \times\left(\omega^{\omega}\right)^{l}$, we say that a sequence $\left\{E_{n}\right.$, $n \geq 0\}$ of subsets of $X$ is uniformly in $\Gamma$ if $x \in E_{n}$, as a relation in $n$ and $x$, belongs to $\Gamma$. For unexplained terminology, the reader is referred to [5].

\section{Main Results}

The proof of the multiple separation theorem rests on the

Covering lemma. Suppose $\Gamma$ is a Spector pointclass closed under $\forall^{\omega^{\omega}}$ or $\exists^{\omega^{\omega}}$. Let $N \subseteq P(\omega)-\{\varnothing\}$ be complete, and assume that $N$ belongs to $7 \Gamma$. Let $\eta_{0} \subseteq \omega$ be such that $\eta_{0} \notin N$ and $\eta_{0}$ belongs to $\neg \Gamma$. Then there is $\eta^{*} \subseteq \omega$ such that $\eta_{0} \subseteq \eta^{*}, \eta^{*} \notin N$, and $\eta^{*}$ belongs to $\Delta$.

Proof. If $\eta_{0}$ belongs to $\Gamma$, we can take $\eta^{*}=\eta_{0}$. So we assume that $\eta_{0}$ does not belong to $\Gamma$. Let $\varphi$ be a $\Gamma$-norm on $\omega-\eta_{0}$. Define

$$
\eta_{1}=\left\{m \in \omega:\left\{n: m \leq_{\varphi}^{*} n\right\} \notin N\right\} .
$$

Claim. $\eta_{1}$ belongs to $\Gamma$.

Let us assume the claim and complete the proof of the lemma.

Observe that $\eta_{0} \subseteq \eta_{1}$. For if $m \in \eta_{0}$, then $\left\{n: m \leq_{\varphi}^{*} n\right\}=\varnothing \notin N$, so $m \in \eta_{1}$. Since $\eta_{0} \notin \Gamma$ and $\eta_{1} \in \Gamma$, we can choose $m_{0} \in \eta_{1}-\eta_{0}$. Set

$$
\eta^{*}=\left\{n \in \omega: m_{0} \leq_{\varphi}^{*} n\right\} \text {. }
$$

Then, as is easily checked, $\eta_{0} \subseteq \eta^{*}$ and $\eta^{*} \notin N$ because $m_{0} \in \eta_{1}$. Plainly, $\eta^{*} \in \Gamma$. To see that $\eta^{*} \in 7 \Gamma$ as well, note that

$$
\eta^{*}=\left\{n \in \omega: \neg\left(n<_{\varphi}^{*} m_{0}\right)\right\}
$$

since $m_{0} \notin \eta_{0}$. So $\eta^{*} \in \Delta$ and we are done.

For the claim, assume first that $\Gamma$ is closed under $\forall^{\omega}$. We will now show that

$$
m \in \eta_{1} \leftrightarrow(\forall \eta)\left[(\forall n)\left(n \in \eta \rightarrow \neg\left(n<_{\varphi}^{*} m\right)\right) \rightarrow \eta \notin N\right] .
$$

Equivalence (1) will imply that $\eta_{1} \in \Gamma$. To prove (1), assume that $m \in \eta_{1}$ and that $\eta \subseteq\left\{n \in \omega: \neg\left(n<_{\varphi}^{*} m\right)\right\}$. It is easy to see that

$$
\left\{n \in \omega: \neg\left(n<_{\varphi}^{*} m\right)\right\}= \begin{cases}\eta_{0} & \text { if } m \in \eta_{0}, \\ \left\{n \in \omega: m \leq_{\varphi}^{*} n\right\} & \text { if } m \notin \eta_{0} .\end{cases}
$$


In either case, $\eta$ is contained in a set which does not belong to $N$, so $\eta \notin N$, since $N$ is complete. Conversely, assume that $m \notin \eta_{1}$. Let $\eta=\{n \in \omega$ : $\left.7\left(n<_{\varphi}^{*} m\right)\right\}$, so $\eta=\left\{n \in \omega: m \leq_{\varphi}^{*} n\right\}$ as $m \notin \eta_{0}$. Since $m \notin \eta_{1}$, it follows that $\eta \in N$. This establishes (1).

Next, assume that $\Gamma$ is closed under $\exists^{\omega^{\omega}}$. We will now show that

$$
m \in \eta_{1} \leftrightarrow(\exists \eta)\left[\eta \notin N \&(\forall n)\left(\neg\left(n<_{\varphi}^{*} m\right) \rightarrow n \in \eta\right)\right] .
$$

Equivalence (3) will imply that $\eta_{1} \in \Gamma$. To prove (3), let $m \in \eta_{1}$. Set $\eta=$ $\left\{n \in \omega: \neg\left(n<<_{\varphi}^{*} m\right)\right\}$. By (2), $\eta \notin N$, which proves the right-hand side of (3). Conversely, suppose $m \notin \eta_{1}$ and let $\eta \subseteq \omega$ be such that $\{n \in \omega$ : $\left.\neg\left(n<<_{\varphi}^{*} m\right)\right\} \subseteq \eta$. It follows that $\left\{n \in \omega: m \leq_{\varphi}^{*} n\right\} \subseteq \eta$; hence, $\eta \in N$ as $N$ is complete. This establishes (3) and the proof is complete.

We are now ready to prove an effective version of the multiple separation theorem for duals of Spector pointclasses. Statement (a) in the theorem is known in the classical literature as the multiple second principle of separation, while statement (b) is called the multiple first principle of separation.

Multiple separation theorem. Suppose $\Gamma$ is a Spector pointclass closed under $\forall^{\omega^{\omega}}$ or $\exists^{\omega^{\omega}}$. Let $N$ be a complete nonempty subset of $P(\omega)-\{\varnothing\}$, and assume that $N$ belongs to $\neg \Gamma$. Let $\left\{E_{n}, n \geq 0\right\}$ be a sequence of subsets of $X$ which is uniformly in $7 \Gamma$.

(a) Then there is a sequence $\left\{C_{n}, n \geq 0\right\}$ of subsets of $X$ which is uniformly in $\Gamma$ and such that $E_{n}-\Phi_{N}\left(E_{0}, E_{1}, \ldots\right) \subseteq C_{n}$ for every $n \geq 0$, and $\Phi_{N}\left(C_{0}, C_{1}, \ldots\right)=\varnothing$.

(b) If $\Phi_{N}\left(E_{0}, E_{1}, \ldots\right)=\varnothing$, then there is a sequence $\left\{B_{n}, n \geq 0\right\}$ of subsets of $X$ which is uniformly in $\Delta$ and such that $E_{n} \subseteq B_{n}$ for every $n \geq 0$, and $\Phi_{N}\left(B_{0}, B_{1}, \ldots\right)=\varnothing$.

Proof. Set $E=\Phi_{N}\left(E_{0}, E_{1}, \ldots\right)$. Next, observe that

$$
x \notin E \leftrightarrow(\exists \eta \in \Delta(x))\left[\eta \notin N \&(\forall n)\left(x \in E_{n} \rightarrow n \in \eta\right)\right] .
$$

The implication ' $\leftarrow$ ' in (4) follows immediately from the definition of $E$ and the fact that $N$ is complete. For the reverse implication, let $x \notin E$. Set $\eta_{0}=\left\{n \in \omega: x \in E_{n}\right\}$ so that $\eta_{0}$ belongs to $\neg \Gamma(x)$ and $\eta_{0} \notin N$. By the Covering lemma relativized to $x$, there is $\eta$ in $\Delta(x)$ such that $\eta \notin N$ and $\eta_{0} \subseteq \eta$. This $\eta$ witnesses the right-hand side of (4).

It now follows from (4) and the theorem on restricted quantification [5, 4D.3] that $X-E$ belongs to $\Gamma$. Furthermore, by the strong $\Delta$-selection principle [5, 4D.6], there is a $\Gamma$-recursive partial function $f: X \rightarrow P(\omega)$ such that

$$
f(x) \downarrow \leftrightarrow x \in X-E
$$

and

$$
x \in X-E \rightarrow f(x) \notin N \&(\forall n)\left(x \in E_{n} \rightarrow n \in f(x)\right) .
$$

Define

$$
R(x, n) \leftrightarrow x \notin E \& n \in f(x) .
$$

Plainly, $R$ is in $\Gamma$. Set $C_{n}=\{x \in X: R(x, n)\}, n \geq 0$, so that the sequence $\left\{C_{n}, n \geq 0\right\}$ is uniformly in $\Gamma$. If $x \in E_{n}-E$, then $R(x, n)$, so $x \in C_{n}$. Suppose next that $x \in \Phi_{N}\left(C_{0}, C_{1}, \ldots\right)$. It follows that $\left\{n \in \omega: x \in C_{n}\right\}=$ 
$\{n \in \omega: R(x, n)\}$ belongs to $N$, since $N$ is complete. Hence, the set $\{n \in$ $\omega: R(x, n)\}$ is nonempty, so $x \notin E$. It follows that $f(x) \downarrow$ and, since then $f(x)=\{n \in \omega: R(x, n)\}, f(x)$ must belong to $N$, yielding a contradiction. So $\Phi_{N}\left(C_{0}, C_{1}, \ldots\right)=\varnothing$. This completes the proof of $(\mathrm{a})$.

To prove (b), assume that $E=\varnothing$. Let $S(x, n) \leftrightarrow x \in E_{n}$, so $S$ is in $7 \Gamma$. As $E=\varnothing$, it follows that $S(x, n) \rightarrow R(x, n)$. Since $\Gamma$ is normed, $\neg \Gamma$ has the separation property [5, 4B.10, 4B.11]. It follows that there is a relation $Q$ in $\Delta$ such that

$$
S(x, n) \rightarrow Q(x, n) \text { and } Q(x, n) \rightarrow R(x, n) .
$$

Set $B_{n}=\{x \in X: Q(x, n)\}, n \geq 0$. Then the sequence $\left\{B_{n}, n \geq 0\right\}$ is uniformly in $\Delta$ and $E_{n} \subseteq B_{n}$ for every $n \geq 0$. Moreover, $\Phi_{N}\left(B_{0}, B_{1}, \ldots\right)=$ $\varnothing$ since $B_{n} \subseteq C_{n}$ for every $n \geq 0$. This proves (b).

Remark. The condition in the theorem above that $N$ be complete is not restrictive when $\Gamma$ is closed under $\forall^{\omega^{\omega}}$. For then $N$ can be replaced by $\bar{N}=$ $\Phi_{N}\left(D_{0}, D_{1}, \ldots\right)$ and $\bar{N}$ belongs to $7 \Gamma$ since $N$ does and $7 \Gamma$ is closed under $\exists^{\omega^{\omega}}$. More generally, if $\neg \Gamma$ is closed under $\Phi_{N}$, then $N$ can be replaced by $\bar{N}$.

As mentioned in the introduction, the classical multiple separation theorems at the first and second levels of the projective hierarchy are now immediate consequences of the bold-face version of our result by taking $\Gamma=\Pi_{1}^{1}$ or $\Sigma_{2}^{1}$ [5, p. 207]. Under Projective Determinacy, $\Pi_{n}^{1}$ ( $n$ odd) and $\Sigma_{k}^{1}$ ( $k$ even) are Spector pointclasses [5, 6B.2] and so the bold-face version of our result will yield multiple separation theorems for $\Sigma_{n}^{1}(n$ odd $)$ and $\Pi_{k}^{1}(k$ even $)$.

\section{REFERENCES}

1. R. O. Davies, J. E. Jayne, A. J. Ostaszewski, and C. A. Rogers, Theorems of Novikov type, Mathematika 24 (1977), 97-114.

2. L. Kantorovich and E. Livenson, Memoir on the analytical operations and projective sets (I), Fund. Math. 18 (1932), 214-279.

3. K. Kuratowski, Topology, Vol. I, Academic Press, New York, 1966.

4. A. Liapunov, Séparabilité multiple pour le cas des opérations $\delta s$, C. R. Acad. Sci. U.R.S.S. 53 (1946), 395-398.

5. Y. N. Moschovakis, Descriptive set theory, North-Holland, Amsterdam, 1980.

6. J. Saint-Pierre, Séparation simultanée d'un ensemble analytique de suites d'ensembles $\mathscr{F}$-sousliniens, C. R. Acad. Sci. Paris 285 (1977), 933-936.

School of Statistics, University of Minnesota, Minneapolis, Minnesota 55455

Current address: Department of Statistics, University of California, Berkeley, California 94720 